\title{
Anesthetic Management During Stenting of Obstructed Total Anomalous Pulmonary Return in a Neonate With Single Ventricle Anatomy
}

\author{
Mumin Hakim, c, Mineto Kamata ${ }^{\mathrm{a}}$, Ralph Beltran ${ }^{\mathrm{a}, \mathrm{b}}$, Marco Corridore , $^{\mathrm{a}}$, \\ Joseph D. Tobias ${ }^{\mathrm{a}, \mathrm{b}}$
}

\begin{abstract}
Total anomalous pulmonary venous return (TAPVR) is a relatively rare congenital cardiac abnormality accounting for $1.5-3 \%$ of cases of congenital heart disease (CHD). Despite advances in surgical technique, surgical repair of TAPVR continues to be associated with a significant incidence of perioperative mortality. Morbidity and mortality associated with surgical repair of TAPVR increase dramatically when there are other associated cardiac defects, particularly single ventricle anatomy. We present a neonate with obstructed TAPVR and an unbalanced atrioventricular canal defect with hypoplastic left ventricle who presented for an interventional cardiac catheterization procedure (stenting of an obstructed vertical vein). The anesthetic management of such patients is discussed and options for intraoperative care are presented.
\end{abstract}

Keywords: Vertical vein; Total anomalous pulmonary venous return; Congenital heart defect; Hypoplastic left heart syndrome

\section{Introduction}

Total anomalous pulmonary venous return (TAPVR) is a relatively rare type of congenital heart disease (CHD) accounting for $1.5-3 \%$ of such defects $[1,2]$. Failure of the formation of a common pulmonary vein from the left atrium with persistent connection of the primitive pulmonary vascular plexus to the common cardinal and umbilico-vitelline veins results in TAPVR $[3,4]$. TAPVR is classified based on the site of pulmonary venous drainage including supracardiac (40-50\%), intracardiac (15-20\%), infracardiac (25-30\%) or mixed type

Manuscript accepted for publication January 11, 2017

aDepartment of Anesthesiology \& Pain Medicine, Nationwide Children's Hospital, Columbus, $\mathrm{OH}$, USA

${ }^{b}$ Department of Anesthesiology \& Pain Medicine, The Ohio State University College of Medicine, Columbus, OH, USA

${ }^{\mathrm{c} C}$ Corresponding Author: Mumin Hakim, Department of Anesthesiology \& Pain Medicine, Nationwide Children's Hospital, 700 Children's Drive, Columbus, OH, USA. Email: Mumim.Hakim@Nationwidechildrens.org

doi: https://doi.org/10.14740/jmc2724w
$(5-10 \%)[3,4]$. A second consideration is whether the venous return is obstructed or non-obstructed. Obstruction may result from external compression from surrounding structures, or reduced caliber of the internal lumen due to pulmonary vein hypoplasia or stenosis. Neonates and infants with obstructed TAPVR present with severe cyanosis, pulmonary hypertension, and low cardiac output requiring emergent intervention [4-6]. The therapeutic approach is defined by identifying the drainage sites and the level of obstruction.

Morbidity and mortality associated with TAPVR repair increase dramatically when another cardiac anomaly is present, particularly when combined with single ventricle anatomy [2]. The anesthetic management of either obstructed TAPVR or an unbalanced atrioventricular canal (AVC) with left ventricle hypoplasia can be quite challenging. Each anomaly carries its own risks and unique anesthetic management goals. We present a neonate with obstructed TAPVR, and unbalanced atrioventricular (AV) defect with hypoplastic left heart syndrome (HLHS), who presented for stenting of an obstructed vertical vein in the cardiac catheterization suite. The anesthetic management of such patients is discussed and options for intraoperative care are presented.

\section{Case Report}

Institutional Review Board (IRB) approval is not required for single patient case reports at Nationwide Children's Hospital (Columbus, $\mathrm{OH}$ ). The patient was a $3.5 \mathrm{~kg}$, term neonate who presented on day of life (DOL) 1 to the cardiac catheterization suite for stenting of obstructed TAPVR. She had been diagnosed in utero with TAPVR and unbalanced AVC with a hypoplastic left ventricle. The plan was to palliate the lesion in a two-stage procedure in an attempt to minimize the perioperative risks. The first step was to open the obstructed supracardiac pulmonary venous return by placing a stent in the vertical vein in the cardiac catheterization suite. Following recovery from this procedure with restoration of pulmonary venous return, and resolution of pulmonary hypertension, a standard hybrid procedure for hypoplastic left ventricle was planned [7].

The patient was born at 38 weeks gestation via spontaneous vaginal delivery at an outside hospital. Her APGAR scores were 4 and 6 . Her trachea was intubated with a $3.5 \mathrm{~mm}$ uncuffed endotracheal tube, and she transferred to our institution 
for further care. Initial echocardiogram showed aortic atresia, hypoplastic left ventricle, ascending aorta and transverse aortic arch, supracardiac TAPVR with obstruction, unbalanced AVC, qualitatively normal ventricular function, large patent foramen ovale, and patent ductus arteriosus (PDA). In the neonatal intensive care unit, room air oxygen saturation $\left(\mathrm{SpO}_{2}\right)$ was $50-60 \%$, and her blood pressure (BP) was 50 - 60/30 - 40 $\mathrm{mm} \mathrm{Hg}$. Umbilical arterial and venous catheters were placed, and prostaglandin $\mathrm{E}_{1}$ (PGE) infusion was started at $0.05 \mu \mathrm{g} /$ $\mathrm{kg} / \mathrm{min}$. Additionally, a dobutamine infusion was initiated at 5 $\mu \mathrm{g} / \mathrm{kg} / \mathrm{min}$ to support hemodynamic function. The initial chest radiograph showed significant pulmonary venous congestion and pulmonary edema. Arterial blood gas analysis (ABG) revealed hemoglobin $15.2 \mathrm{~g} / \mathrm{dL}, \mathrm{pH} 7.35, \mathrm{PaCO}_{2} 47 \mathrm{~mm} \mathrm{Hg}$, $\mathrm{PaO}_{2} 23 \mathrm{~mm} \mathrm{Hg}$, and $\mathrm{HCO}_{3} 27 \mathrm{~mm} \mathrm{Hg}$. Her ventilator settings were inspired oxygen $\left(\mathrm{FiO}_{2}\right)$ concentration $30 \%$, peak inspiratory pressure $26 \mathrm{~cm} \mathrm{H}_{2} \mathrm{O}$, positive end-expiratory pressure (PEEP) $6 \mathrm{~cm} \mathrm{H} \mathrm{H}_{2} \mathrm{O}$, and rate 26 breaths/min. The patient was subsequently transported urgently to the goal of stenting the obstructed vertical vein. In the catheterization suite, routine American Society of Anesthesiologists' monitors were placed including a pre-ductal blood pressure cuff and pulse oximeter. Invasive blood pressure was monitored from the umbilical artery catheter. Baseline vital signs revealed a heart rate of 165 beats/min, arterial blood pressure of $85 / 60 \mathrm{~mm} \mathrm{Hg}$, and $\mathrm{SpO}_{2}$ $67 \%$ with an $\mathrm{FiO}_{2}$ of $30 \%$. Anesthesia was induced with fentanyl $2 \mu \mathrm{g} / \mathrm{kg}$, maintained with sevoflurane expired concentration $1-3 \%$, and an inspired oxygen concentration of $21 \%$. Neuromuscular blockade was provided with pancuronium. A 22-gauge peripheral intravenous cannula was placed in the left hand. Ventilation was adjusted to maintain normocarbia and fluid management guided by hemodynamic status. Approximately $15 \mathrm{~min}$ after the induction of anesthesia, the mean arterial pressure (MAP) decreased to $30-32 \mathrm{~mm} \mathrm{Hg}$. A $5 \mathrm{~mL} / \mathrm{kg}$ bolus of 5\% albumin was administered with minimal results, but the MAP returned to baseline following a bolus dose of epinephrine $1 \mu \mathrm{g} / \mathrm{kg}$. Subsequent $\mathrm{ABG}$ revealed $\mathrm{pH}$ 7.26, $\mathrm{PaCO}_{2}$ $55 \mathrm{~mm} \mathrm{Hg}, \mathrm{PaO}_{2} 28 \mathrm{~mm} \mathrm{Hg}, \mathrm{HCO}_{3} 25 \mathrm{~mm} \mathrm{Hg}$, base excess (BE) -3.0, hemoglobin $15.6 \mathrm{gm} / \mathrm{dL}$, and ionized calcium 1.41 $\mathrm{mmol} / \mathrm{L}$. Despite adjustments in ventilation and an additional bolus of albumin, the MAP decreased again. The dobutamine infusion was increased to $10 \mu \mathrm{g} / \mathrm{kg} / \mathrm{min}$ after administering a second bolus dose of epinephrine $1 \mu \mathrm{g} / \mathrm{kg}$ which stabilized the MAP at $35-40 \mathrm{~mm} \mathrm{Hg}$. Access for the catheterization was obtained via the right femoral vein. Angiography revealed that the four pulmonary veins entered a common confluence. A dilated confluence of the pulmonary veins emptied into a severely stenotic ( $2 \mathrm{~mm}$ diameter) left vertical which entered the left innominate vein. Stenting the vertical vein proved challenging as hypotension and worsening hypoxemia ensued following catheter placement in the vertical vein. Ultimately, a $28 \mathrm{~mm}$ long multi-link ultra coronary stent was placed in the stenotic vertical vein. The stent initially did not fully dilate the area of stenosis, and multiple passes with a dilating balloon were required. Subsequently, a high pressure balloon had to be used to open the area of stenosis to $5 \mathrm{~mm}$, and reduce the pressure gradient to $2 \mathrm{~mm} \mathrm{Hg}$. Angiography revealed no extravasation or aneurysm formation. Due to the crossing of the vertical vein at the level of the left main bronchus, a bron- choscopy was also performed at the end of the procedure to ensure that the stent was not impinging on this structure. Upon completion of the procedure, the hemoglobin was $9.9 \mathrm{~g} / \mathrm{dL}$ and $20 \mathrm{~mL} / \mathrm{kg}$ of packed red blood cells were transfused. Prior to transfusion, ABG revealed $\mathrm{pH} 7.30, \mathrm{PaCO}_{2} 43 \mathrm{~mm} \mathrm{Hg}, \mathrm{PaO}_{2}$ $25 \mathrm{~mm} \mathrm{Hg}$, and $\mathrm{HCO}_{3} 19 \mathrm{~mm} \mathrm{Hg}$, with a BE of -2.0. Following transfusion, the patient was transported to the cardiac intensive care unit (CTICU) with the trachea intubated and ventilation controlled. Admission vital signs included an $\mathrm{SaO}_{2}$ of $70-80 \%$ and a BP of $70-80 / 40-50 \mathrm{~mm} \mathrm{Hg}$. Dobutamine was weaned to off over the next $24 \mathrm{~h}$ and diuresis initiated. On DOL 5, the patient was returned to the cardiac catheterization suite for the hybrid procedure. The latter included a median sternotomy with placement of bilateral pulmonary arterial bands and an invasive cardiac catheterization procedure with placement of a stent in the ductus arteriosus. Anesthesia for this procedure was induced with fentanyl $2 \mu \mathrm{g} / \mathrm{kg}$, and maintained with sevoflurane using an inspired oxygen concentration of $21 \%$. Neuromuscular blockade was provided by intermittent doses of rocuronium. Ventilation was adjusted to maintain normocarbia and fluid management guided by hemodynamic status. Intraoperatively, the BP varied from 70 - 90 to 40 - $50 \mathrm{~mm} \mathrm{Hg}$ with a heart rate of $140-170$ beats/min. The $2.5 \mathrm{~h}$ procedure was well tolerated and she returned to the CTICU without needing vasoactive agents. The evening following the procedure, ventricular dysfunction developed, and an epinephrine infusion was started and required for $72 \mathrm{~h}$. The patient's trachea was extubated to continuous positive airway pressure (CPAP) on DOL 11 and she was transferred from CTICU to the inpatient cardiology ward on DOL 14. During this time, her hospital course was complicated by feeding difficulties and the development of a nosocomial pneumonia. By DOL 22, there was worsening tachypnea and tachycardia which was attributed to an increasing gradient in the vertical vein, as well as moderate AV regurgitation. She was taken back to the cardiac catheterization suite where the gradient was noted to be $11 \mathrm{~mm} \mathrm{Hg}$. A second stent was placed, yielding elimination of the previous gradient. Following this intervention, her hospital course continued to be complicated with issues regarding feeding intolerance due to diagnosed malrotation, prompting support therapy with parenteral nutrition. Over the ensuing $1-2$ months, she developed restenosis within the stent in the vertical vein, requiring an additional procedure in the cardiac catheterizations suite. At this point she continued to have respiratory insufficiency requiring non-invasive support with CPAP. Given her recurrent and persistent stenosis of the vertical vein despite multiple interventions, a decision was made to proceed early to a comprehensive stage II procedure, and to repair her total anomalous pulmonary venous return [8]. On DOL 124, a second stage palliation was performed. This procedure included repair of TAPVR with a pericardial well and repair of the AV valve. Cardiopulmonary bypass time was $343 \mathrm{~min}$ with a cross clamp time of $121 \mathrm{~min}$, and a circulatory arrest time of $2 \mathrm{~min}$. Toward the conclusion of surgery, hemodynamic and respiratory instability ensued requiring support with continuous infusions of epinephrine, milrinone, and inhaled nitric oxide. She was then transported to the CTICU with an open chest. Postoperative echocardiogram revealed moderate AV valve regurgitation and depressed ventricular function. Progressive 
hemodynamic and respiratory instability resulted in the need for mechanical support with extracorporeal membrane oxygenation (ECMO). She was taken to the cardiac catheterization suite where no anatomical concerns were noted and no additional intervention was provided. Given the patient's declining status, parental decision not to proceed to cardiac transplantation, and continued deterioration, the decision was made to stop all supportive measures. Adequate sedation was provided to ensure comfort, and ECMO support was discontinued. The patient expired without distress with family members at the bedside.

\section{Discussion}

TAPVR remains one of the most challenging CHDs in newborns and young infants. The surgical results of TAPVR repair have improved significantly in the modern era as a result of earlier diagnosis and treatment, improvements in surgical technique, cardiac anesthesia, neonatal myocardial preservation, and postoperative care [3, 9]. However, increased mortality occurs in patients with obstructed TAPVR, associated CHD, and preoperative decompensation with the need for emergency intervention. All three elements described were present in our patient.

When dealing with the association of two different types of CHD, the perioperative hemodynamic and respiratory management must take into account the goals for both lesions. Although the infant with an unbalanced AV canal and HLHS can generally be medically managed and supported with PGE to maintain ductal patency with a non-emergent path to operative repair, the presence of obstructed TAPVR is considered a surgical emergency. The care of such patients is frequently complicated by end-organ effects of obstruction to pulmonary venous return resulting in a critically ill neonate with hemodynamic and respiratory instability. The obstruction to pulmonary venous return can lead to pulmonary edema, overt right heart failure with pulmonary hypertension, profound hypoxemia, and hemodynamic instability. Prior to surgical or cardiac catheterization intervention, the goals of anesthetic management include effective ventilation with correction of hypoxemia and hypercarbia, control of pulmonary vascular resistance (PVR), correction of acid/base and electrolyte imbalances, and the maintenance of hemodynamic stability with inotropic support. Since medical therapy will have limited impact without surgical intervention or cardiac catheterization, patients should be urgently optimized for a potential intervention.

In patients with HLHS, a PDA is essential for maintenance of systemic blood flow. Patency of the PDA is accomplished with prostaglandin administration. However, this results in a situation in which the ratio between pulmonary and systemic blood flow (Qp/Qs) is determined by the difference between the systemic and pulmonary vascular resistance. The ideal Qp/ Qs ratio of 1:1 is identified clinically by a systemic oxygen saturation of $75 \%$. As the pulmonary blood flow increases, the systemic oxygen saturation increases. As the Qp/Qs ratio exceeds $1.5-2: 1$, there are signs of pulmonary over-circulation including pulmonary edema and inadequate tissue oxygen delivery, prompting the development of metabolic acidosis despite an increasing systemic oxygen saturation [10]. In the setting of obstruction to pulmonary venous return, over-flow of the pulmonary circulation can lead to a rapid deterioration in respiratory function.

The care of these critically ill neonates with CHD is compounded by the fact that direct measurements of cardiac output are not possible. Intraoperative data have demonstrated a progressive increase in the base deficit when the $\mathrm{PaO}_{2}$ drops below $50 \mathrm{~mm} \mathrm{Hg}$ [11]. Conversely, an increased SVR can generate lactic acidosis and a low cardiac output state due to high afterload. With single ventricle physiology, maintenance of systemic oxygen delivery is dependent on optimizing cardiac output and arterial oxygen content. Optimal cardiac output requires attention to volume status and specifically preload, vascular resistance or afterload, heart rate, rhythm, and myocardial contractility. Arterial oxygen content is predominately dependent on hemoglobin content. Simple intraoperative maneuvers to maintain the Qp/Qs ratio include adjusting the inspired oxygen concentration to maintain an oxygen saturation of $75-80 \%$, maintaining normocarbia, a normal $\mathrm{pH}$, and normothermia.

In single ventricle physiology, myocardial function may be compromised due to chronic volume overload, a decreased ability of the right ventricle to sustain systemic pressures, thus potentially leading to myocardial ischemia. Myocardial function therefore should be assessed preoperatively via echocardiographic examination. Vasoactive agents such as milrinone, dobutamine, or low dose epinephrine, are chosen to improve inotropy while decreasing SVR. When dealing with systemic hypotension, particularly in the face of concomitantly increasing PVR, vasopressin may be an effective option for reversing these trends while maintaining MAP and perfusion [12].

Non-invasive monitoring of tissue oxygenation using near infrared spectroscopy may provide an early warning of decreased oxygen delivery [13]. As noted in our patient, invasive monitoring is warranted in patients with depressed ventricular function. This strategy provides not only access for invasive blood pressure monitoring, but also access for arterial blood gas, ionized calcium, and lactic acid sampling. Close followup of these values may be useful to determine acid-base status, and $\mathrm{PaCO}_{2}$ in a setting where control of these variables is mandatory to optimize myocardial function and Qp/Qs ratio.

One of the problems when there is more than one associated CHD lesion is that the anesthetic and medical therapies of one condition may directly hinder care of the other. For this patient with coexisting obstructed TAPVR and unbalanced AVC defect, we provide an example of how the hemodynamic goals of these two lesions may directly oppose each other. For instance, although control of PVR with increased oxygenation, mild hypocarbia, and correction of acidosis is needed for the patient with diagnosis of TAPVR with associated pulmonary hypertension, lowering PVR may result in excessive pulmonary blood flow, decreased systemic oxygen delivery, and decreased coronary artery perfusion in the setting of HLHS. In many ways, this case is similar to management of an infant with HLHS with an intact atrial septum, except the obstructed vertical vein represents a more dynamic component as opposed to a fixed obstruction. For these lesions, the primary 
intervention requires relief of the obstruction to pulmonary venous return by either a surgical or cardiac catheterization procedure $[14,15]$. Medical therapy and stabilization will provide only temporary and limited reversal of the patient's compromised status. As illustrated in this case, these infants require immediate and emergent interventions to relieve the anatomic obstruction to pulmonary venous return. Following these interventions, a balance must be achieved between treatment of pulmonary hypertension, and optimizing systemic cardiac output while maintaining adequate oxygen saturation.

When dealing with two coexisting and physiologically opposed types of CHD as it did in our patient, the hemodynamic and respiratory management must take into account the supportive therapeutic goals for both lesions. This strategy includes a comprehensive medical and surgical approach with the primary goal of facilitating emergent surgical intervention. To this end, it is essential to use effective ventilation techniques with the aim of correcting hypoxemia and hypercarbia in an effort to clinically manage PVR. Also important imperative is to correct problems with acid/base, electrolyte imbalance and to maintain hemodynamic stability using inotropic support as indicated. Once the anatomic obstruction to unimpeded pulmonary venous return has been relieved, ongoing care is predominantly dictated by the balance of the Qp/Qs ratio in the setting of HLHS. However, patients with HLHS may require therapy aimed at lowering PVR, because pulmonary hypertension is frequently present in the setting of TAPVR even after the obstruction has been relieved. Maintenance of systemic oxygen delivery is dependent on optimizing cardiac output and arterial oxygen content. Control of the Qp/Qs is achieved by adjusting the inspired oxygen concentration to maintain an oxygen saturation of $75-80 \%$, maintaining normocarbia, normal $\mathrm{pH}$, and normothermia. Anesthetic agents such as fentanyl are chosen and adjusted to minimize their effects on myocardial performance. Given the complex nature of such patients, the key to a successful outcome is the close cooperation of a multi-disciplinary pediatric team including intensivists, anesthesiologists, cardiologists, and cardiothoracic surgeons.

\section{Grant Support}

None.

\section{Conflicts of Interest}

None.

\section{References}

1. Michielon G, Di Donato RM, Pasquini L, Giannico S, Brancaccio G, Mazzera E, Squitieri C, et al. Total anomalous pulmonary venous connection: long-term appraisal with evolving technical solutions. Eur J Cardiothorac Surg. 2002;22(2):184-191.

2. Karamlou T, Gurofsky R, Al Sukhni E, Coles JG, Wil- liams WG, Caldarone CA, Van Arsdell GS, et al. Factors associated with mortality and reoperation in 377 children with total anomalous pulmonary venous connection. Circulation. 2007;115(12):1591-1598.

3. Sano S, Brawn WJ, Mee RB. Total anomalous pulmonary venous drainage. J Thorac Cardiovasc Surg. 1989;97(6):886-892.

4. Wang JK, Lue HC, Wu MH, Young ML, Wu FF, Wu JM. Obstructed total anomalous pulmonary venous connection. Pediatr Cardiol. 1993;14(1):28-32.

5. Seale AN, Uemura H, Webber SA, Partridge J, Roughton M, Ho SY, McCarthy KP, et al. Total anomalous pulmonary venous connection: morphology and outcome from an international population-based study. Circulation. 2010;122(25):2718-2726.

6. Munsi AS, Hussain M, Rima R, Biswas R, Sayeed A. Clinical profile of patients with total anomalous pulmonary venous return and their short term outcome in pediatric cardiac centre at Dhaka Shishu Hospital. Bangl J Med Sci. 2015;14(3):270-273.

7. Galantowicz M, Cheatham JP, Phillips A, Cua CL, Hoffman TM, Hill SL, Rodeman R. Hybrid approach for hypoplastic left heart syndrome: intermediate results after the learning curve. Ann Thorac Surg. 2008;85(6):20632070; discussion 2070-2061.

8. Galantowicz M, Yates AR. Improved outcomes with the comprehensive stage 2 procedure after an initial hybrid stage 1. J Thorac Cardiovasc Surg. 2016;151(2):424-429.

9. Kelle AM, Backer CL, Gossett JG, Kaushal S, Mavroudis C. Total anomalous pulmonary venous connection: results of surgical repair of 100 patients at a single institution. J Thorac Cardiovasc Surg. 2010;139(6):1387-1394 e1383.

10. Yuki K, DiNardo JA. Comparison of actual oxygen delivery kinetics to those predicted by mathematical modeling following stage 1 palliation just prior to superior cavopulmonary anastomosis. Paediatr Anaesth. 2015;25(2):174179.

11. Hansen DD, Hickey PR. Anesthesia for hypoplastic left heart syndrome: use of high-dose fentanyl in 30 neonates. Anesth Analg. 1986;65(2):127-132.

12. Siehr SL, Feinstein JA, Yang W, Peng LF, Ogawa MT, Ramamoorthy C. Hemodynamic Effects of Phenylephrine, Vasopressin, and Epinephrine in Children With Pulmonary Hypertension: A Pilot Study. Pediatr Crit Care Med. 2016;17(5):428-437.

13. Tobias JD. Cerebral oxygenation monitoring: nearinfrared spectroscopy. Expert Rev Med Devices. 2006;3(2):235-243.

14. Schranz D, Jux C, Akintuerk H. Novel catheter-interventional strategy for intracardiac connecting of total anomalous pulmonary venous return in newborns with hypoplastic left heart-syndrome prior to hybrid approach. Catheter Cardiovasc Interv. 2013;82(4):564-568.

15. Kobayashi D, Forbes TJ, Aggarwal S. Palliative stent placement in vertical vein in a $1.4 \mathrm{~kg}$ infant with obstructed supracardiac total anomalous pulmonary venous connection. Catheter Cardiovasc Interv. 2013;82(4):574-580. 